\title{
Haptic-enabled collaborative learning in virtual reality for schools
}

\author{
Mary Webb ${ }^{1}$ Megan Tracey ${ }^{1}$. William Harwin ${ }^{2}$. Ozan Tokatli ${ }^{2}$. \\ Faustina Hwang ${ }^{2} \cdot$ Ros Johnson ${ }^{3} \cdot$ Natasha Barrett $^{2} \cdot$ Chris Jones $^{2}$
}

Received: 20 April 2021 / Accepted: 16 June 2021 / Published online: 2 July 2021

(c) The Author(s) 2021

\begin{abstract}
This paper reports on a study which designed and developed a multi-fingered haptic interface in conjunction with a three-dimensional (3D) virtual model of a section of the cell membrane in order to enable students to work collaboratively to learn cell biology. Furthermore, the study investigated whether the addition of haptic feedback to the 3D virtual reality (VR) simulation affected learning of key concepts in nanoscale cell biology for students aged 12 to 13 . The haptic interface was designed so that the haptic feedback could be turned on or switched off. Students $(N=64)$, in two secondary schools, worked in pairs, on activities designed to support learning of specific difficult concepts. Findings from observation of the activities and interviews revealed that students believed that being immersed in the $3 \mathrm{D}$ VR environment and being able to feel structures and movements within the model and work collaboratively assisted their learning. More specifically, the pilot/co-pilot model that we developed was successful for enabling collaborative learning and reducing the isolating effects of immersion with a 3D headset. Results of pre and post-tests of conceptual knowledge showed significant knowledge gains but addition of haptic feedback did not affect the knowledge gains significantly. The study enabled identification of important issues to consider when designing and using haptic-enabled 3D VR environments for collaborative learning.
\end{abstract}

Keywords Haptics · Haptic feedback · Virtual reality $\cdot 3 \mathrm{D} \cdot$ Cell biology · Collaborative learning $\cdot$ Nanoscale $\cdot$ Collaborative roles

Mary Webb

mary.webb@kcl.ac.uk

1 School of Education, Communication and Society, King's College London, Franklin-Wilkins Building - Waterloo Bridge Wing, Waterloo Rd., London SE1 9NN, UK

2 School of Biological Sciences, University of Reading, Reading, UK

3 The Abbey, Reading, UK 


\section{Introduction and background}

The term haptic is often used to refer both to a sensory perception and to human machine interfaces. In the former sense it relates to the integration of touch and proprioceptive cues, with other sensory information (Gibson \& Carmichael, 1966; Lederman \& Klatzky, 2009). In the later sense, a haptic interface can apply forces and vibrations to the individual to increase the user's awareness of the interaction. Availability and capability of haptic technology is increasing but the importance of touch feedback for learning is as yet unclear (Zacharia, 2015). Furthermore, design considerations and guidelines for multisensory learning systems remain challenging and underspecified (Seifi et al., 2020). More fundamentally, although there is general acceptance that touch plays an important role in exploring the world around us, the importance of haptic experiences and haptic feedback for learning has been under-researched compared with visual and auditory (Gallace $\&$ Spence, 2009). To date, the use of haptics technology has been limited mostly to surgical, dental and veterinary simulations due to the high cost of the devices, and in these fields they are used primarily to train and test surgical visual-motor skills. Furthermore, while haptic feedback interactions between individual learners and technology have been shown to be beneficial for the development of skills (San Diego et al., 2012), research into whether haptic feedback may be beneficial for developing understanding and memory retention is much more limited.

There are many areas of learning especially in science and technology but also in history and geography where virtual reality simulations can enable learners to experience phenomena that are difficult to explore in real life. There is some evidence that dynamic computer-based visualisations can support the development of understanding (McElhaney et al., 2015; Rundgren \& Tibell, 2010). Currently such systems can provide visual and auditory experiences but, in future, opportunities are likely to increase for the addition of haptic experiences to virtual reality simulations. (Webb et al., 2017) argued that the potential benefits, for learning science concepts, of the addition of haptic feedback to a 3D VR simulation derive from: 1) the known general benefits of multisensory learning compared with uni-sensory (Shams \& Seitz, 2008); 2) engagement and motivational effects of a more realistic experience; and 3 ) the more specific possibility that haptic feedback interaction will support the mental visualisation that is necessary for understanding many key processes in science (Gilbert et al., 2007; Rundgren \& Tibell, 2010; Tuckey \& Selvaratnam, 1993). However, there is also the possibility that providing additional stimuli might confuse or distract learners or create technical difficulties that detract from the learning experience.

This paper is based on a study that aimed to examine the value of haptic feedback to support the development of conceptual understanding of membrane structure and function in students aged 12-13. This topic was chosen because it is known to be difficult to understand and to teach and students are prone to misconceptions and misunderstanding of both membrane structure and the dynamic processes involved. Furthermore, these dynamic processes are controlled by forces at the nanoscale level that can be simulated with haptic feedback so that 
students can be given opportunities to "feel" the forces. Understanding of complex dynamic systems has not been easy to achieve through the 2-D representations and static 3-D models frequently in use in science classrooms in secondary schools (Gilbert, 2005; Webb, 2008). Moreover, the use of diagrammatic representations, simulations and animations to promote understanding of the dynamic processes of membrane transport has yielded mixed results (see Rundgren \& Tibell, 2010 for a review). We propose that haptic (virtual touch) interaction in a three-dimensional (3-D) virtual reality (VR) simulation may support mental representation and visualisation and the development of understanding.

Our research objectives were to: 1) design and develop a haptic-enabled 3D VR model of the cell membrane for students to explore difficult concepts of membrane structure and function through interactive multisensory collaborative activities; 2) investigate whether or not being able to feel the interactions through haptic feedback affected students' development of understanding of concepts and 3) examine students' perspectives on the interactive learning experience and the value of haptic feedback. In order to achieve (2), we aimed to design an interface in which haptic feedback could be enabled or disabled such that in either condition the user could interact with the model.

In this paper, we first discuss the theoretical framework and previous research. Then we explain the principles and design of the VR environment that we developed for this study and the haptic-enabled interface designed to explore the VR environment. We then discuss the nature of the interactive learning environment and activities that we developed. The methods for collecting data, results and discussion of findings then follow. Finally, we consider the implications for future design and use of haptic-enabled VR environments for learning in schools.

\section{Theoretical framework}

The design of the study was dependent on the relationship between learning and haptic feedback which has been proposed to influence visualization and is known to interact with other modalities. Furthermore, the haptic feedback was designed to work in the context of science simulations existing in 3D virtual reality. Therefore, existing knowledge and theory about the interplay between these elements is discussed in this section in order to shape the theoretical framework for the study.

\subsection{Haptic feedback and learning}

Previous haptic interfaces were typically capable of only a single point of contact supporting a pointer or probe. Recent developments in haptic technology have enabled the deployment of two or more points of contact (Harwin \& Barrow, 2013; Melder \& Harwin, 2004). A key advantage of multi-finger haptics is that they provide a natural method to position and orientate objects in 6 dimensions, three positions, and three orientations. When compared to visual cues that include shadows and stereo vision multi-finger haptic interfaces have been shown to be superior to 
accurate positioning of objects in both position and orientation (McKnight et al., 2005). Multi-finger haptic interfaces have no effect on the speed of task completion (ibid). However, Multi-finger haptics can more closely mimic manipulation in the real world, and users can feel the haptic forces exerted on those objects as they move them (Tokatli et al., 2016). For example, students may experience forces resulting from concentration gradients and suction effects of transporter proteins in a cell membrane as well as examining surfaces of nanoscale particles through virtual touch.

As mentioned earlier, the importance of haptic experiences and haptic feedback for learning has been under-researched compared with visual and auditory, but a recent study (Novak \& Schwan, 2021) provided some support for the possibility that haptic feedback might improve memory retention. Novak and Schwan's study, in the context of exhibitions of animal husbandry, indicated that the participants who had a haptic experience by touching real objects were able to build a stronger mental representation of the exhibited tools. It has long been recognised that the ability to visualise and to manipulate objects in the imagination is a crucial skill for learning science (see for example Gilbert et al., 2007; Rundgren \& Tibell, 2010; Tuckey \& Selvaratnam, 1993; Xu \& Franconeri, 2015). Visualisation, as a mental process, has been characterised as "complicated, multistep manipulations of spatially presented information” (Linn \& Petersen, 1985 P. 1484).

The nature of the mental representations and processes that enable visualisation are not well understood but in visual working memory systems there are known limitations that constrain people's ability to mentally manipulate 3D objects (Oberauer \& Eichenberger, 2013; Xu \& Franconeri, 2015). Possible theoretical foundation for the suggested improved learning associated with haptic feedback for supporting visualisation comes from dual coding theory and its interaction with cognitive load theory. Dual coding theory (Paivio, 1969, 2014) proposes that distinct interconnected systems for different sensory modalities act synergistically, allowing the beneficial effect of the combined coding of sensory information. According to this theory, the addition of the haptic sense would work synergistically with other sensory modes to aid the coding of complex information. Cognitive load theory (Sweller, 1994) suggests that an individual's working memory is put under cognitive load as they process new domain specific concepts and skills. This "intrinsic cognitive load" works additively with "extraneous cognitive load", created by the instructional environment. Since working memory is subject to a maximum cognitive load, the instructional design needs to minimise extraneous cognitive load (Sweller, 2016). It is thought that having another channel of information in a different modality, i.e. haptic, may also help alleviate the cognitive load and aid learning. Students' learning generally depends on retrieving information from long-term memory storage and this prior knowledge does not contribute to their cognitive load (Sweller, 2016). Cognitive load theory has been applied to individual interaction with computer systems (Wong et al., 2012), especially multimedia systems (Anmarkrud et al., 2019; Mayer \& Moreno, 2003) and also in relation to collaborative learning (Kirschner et al., 2009; Kirschner et al., 2018). Kirschner et al. (2009) proposed that when individuals are working collaboratively, not only can they draw on each other's long-term memory stores, but their working memories are pooled thus creating a 
collective working memory. Therefore, the positive effects of collaborative learning in computer-based environments, that have been measured by a recent meta-analysis (Chen et al., 2018), also have theoretical support from cognitive load theory. Both cognitive load theory and dual coding theory are based on the 'modality principle' (Burton \& Sinclair, 2000) which assumes that every modality has its own processing channel within working memory.

In this study an overarching aim was to enable the use of haptic feedback within the context of a 3D VR simulation that would support interactive collaborative learning suitable for a classroom context. Therefore, it was important to consider how the haptic feedback might interact with other modalities. In particular, the visual elements of the 3D VR environment that were interesting and novel for the students might be expected to draw students' attention, thus leading to "visual dominance". Visual dominance is a well-known psychological phenomenon, which suggests that people are more likely to notice and respond to visual stimuli than those from their other senses. For example, even in experiments where participants were compelled to attend to a particular sensory stimulus, an irrelevant visual stimulus interfered much more with their response to an auditory stimulus than vice versa (Lukas et al., 2010). Lukas et al. explained these findings in relation to the theory of directed attention (Posner et al., 1976), which claims that visual stimuli present as relatively weak stimuli compared with other modalities so people tend, as their default situation, to focus their attention toward visual stimuli. More recent evidence from fMRI scans confirms a competitive effect between different sensory modalities although that research has focused on visual compared with auditory rather than haptic (Schmid et al., 2011). Such effects are noticed in conditions of high cognitive load (Broadbent et al., 2018). Studies have shown that the visual dominance effect can be overcome by 'user priming' in which expectations are set so that users compensate for weaker parts of the system's multisensory feedback through using their imagination (Pusch \& Lécuyer, 2011). However, such an effect cannot be attributed to haptic feedback alone. This psychological effect has also been shown to enable a haptic sensation without any physical stimulus; a phenomenon known as "pseudo haptics" (ibid.).

\subsection{Learning with simulations, VR and haptic-enablement}

Much previous research has suggested that computer simulations can support the learning of difficult concepts by secondary school students, and enable hypothesis testing in areas of science learning where direct manipulation of real-world objects is not possible (see for example reviews by Rutten et al., 2012; Webb, 2008). Research into the value of virtual reality environments for simulations has been limited to date but a meta-analysis suggested that there are benefits of virtual reality for learning but there were insufficient studies to examine which pedagogical factors may be important e.g. whether collaborative learning in these environments may be beneficial (Merchant et al., 2014). A systematic literature review (Zacharia, 2015) of studies comparing virtual manipulatives with and without the provision of haptic feedback identified only 11 articles. The studies had small sample sizes and results 
were mixed although the majority of studies reported positive effects of haptic feedback (Zacharia, 2015).

Small-scale studies in tertiary education have found a positive impact of haptic devices on learning in science (Schönborn et al., 2011). An experimental study with 20 postgraduate students using a Phantom haptic device and 3D visual representations to explore protein and ligand molecular binding, found that the haptic condition enabled faster docking times (Schönborn et al., 2011). However, increased understanding was limited to answering only one question concerning the specific binding process at the enzyme's active site and the students in both conditions had difficulties in answering a broader range of questions about molecular binding (Bivall et al., 2011). Jones et al. (2006) experimental study of middle school students $(N=80)$ using desktop 3D stimulations of cell structures found no significant differences in learning gains between the haptic feedback and no-haptic-feedback conditions. A later experimental study (Minogue \& Jones, 2009) of high school students $(N=80)$ using a 3D desktop simulation of membrane permeability, found that those with both visual and haptic feedback achieved a higher level of understanding, measured using the SOLO taxonomy, than students with visual feedback alone. The difference between these two studies suggests that rather than overall learning gains, there may be gains in understanding concepts at a deeper level.

In all of these studies outlined above, students worked individually using haptic devices that allowed only a single point of contact. Evidence for the importance of the type of haptic interface and its sensitivity comes from an experimental study of 36 middle and high school students, working in pairs on a 3D desktop simulation of virus morphology and behaviour (Jones et al., 2006). The study compared haptic devices with differing levels of sensitivity: a low sensitivity haptic joystick, a higher sensitivity Phantom haptic device and a non-haptic mouse as a control. Findings showed that as the sensitivity of the haptic feedback increased, students described more characteristics of the viruses using more haptic terms (ibid.).

In order to support the research objective of enabling collaborative learning in this study, deciding on roles for students was necessary not only to enable the sharing of devices but also to promote interaction. Chen et al's (2018) meta-analysis showed the importance of role assignment with a significant medium effect size in promoting collaborative interaction. More specifically, role assignment raised students' responsibility for group work, awareness of collaboration and improved student learning outcomes (ibid.). In order to support learning of programming, which is also known to be difficult for many students, a paired learning process involving role assignment has been used effectively in schools and shown significant benefits in higher education (Umapathy \& Ritzhaupt, 2017). In this "pair programming" arrangement one of the pair (the pilot) writes the program on the computer while the other (the navigator) provides guidance and feedback and they regularly swap roles. A study by Rodríguez et al (2017) of the interaction during pair programming identified the importance of students' dialogue and particularly of expressing uncertainty which often led to further clarification and ideas.

In summary there is theoretical support for the possibility that haptic feedback may enhance students' learning by reducing cognitive load but other factors to consider were the design of the instructional environment to minimise extraneous 
cognitive load, the specific type of haptic interface, interaction between the modalities during the learning experiences, the assignment of collaborative roles in relation to the capabilities of the system and the students' prior knowledge and experience. Furthermore, a prerequisite for the study was the design of the learning tasks and their focus on specific concepts that presented learning challenges that were likely to be supported by a VR simulation enabled by haptic technology.

\section{Concept development considerations}

In order to focus the design of the system and activities on appropriate concepts, it was necessary to consider not only curriculum requirements but also learners' problems in understanding concepts. Specific issues in understanding membrane function and related concepts in cell biology include: a persistent anthropomorphic view of processes and assignment of intentionality to cell functions (Flores et al., 2003); issues in understanding magnification and scaling (Marsh et al., 2001); problems in understanding randomness in relation to diffusion (Garvin-Doxas \& Klymkowsky, 2008); being able to negotiate meaning when moving between representations at different levels e.g. whole-body, cell, nanoscale (Olander et al., 2018); differences in mental visualisations of cell structures and processes resulting from different visual representations (Rundgren \& Tibell, 2010); and the importance of prior knowledge in interpreting visual representations (Tasker \& Dalton, 2008). Specific misconceptions that have been identified include: molecules diffuse depending on the space available (Tekkaya, 2003); diffusion in a cell depends on the 'living' processes of the cell and therefore stops following the death of the cell (Oztas, 2014); a substance dissolved in a liquid spreads out by breaking into smaller particles.

It is likely that some of these significant student comprehension problems and misconceptions of the functioning of cell membranes result from limited models and representations currently used for teaching (Flores et al., 2003; Malińska et al., 2016). Thus, in the design of the environment, we needed to consider the concepts to be developed, known misconceptions, possible representations of the cell structures and their relationship to existing models with which students were familiar. Based on considerations of the existing curriculum, the following are key concepts to be developed with students aged 12-13:

1. The cell membrane is a barrier to the movement of some substances whereas others pass through freely.

2. Substances move in the cellular fluid by diffusion and some substances are able to continue moving by diffusion through the membrane.

3. The movement of substances that are able to freely diffuse depends on their individual diffusion gradients.

4. The cell membrane is a dynamic structure in which membrane proteins 'float'.

5. Specialised proteins enable the movement of some substances through the membrane either by acting as simple channels or as carrier proteins that bind to specific molecules and change shape as a molecule passes through the membrane (facilitated diffusion). 
This conceptual content would comprise the "intrinsic cognitive load" of the material to be learned whose complexity is apparent in the many interrelationships between the concepts which indicates a high intrinsic cognitive load (Sweller, 2010). All of these concepts involve movement and interaction between elements in the model and therefore students' learning might benefit from haptic feedback to experience the movements and forces involved and potentially to reduce cognitive load as a result of the additional sensory channel. However, most of the movement and interactions could also be observed visually. Exceptions that were not visually observable were the forces due to diffusion gradients and the forces created by binding of molecules with their specific membrane transporter proteins.

\section{Design of the VR environment}

Designing the 3D VR environment to accommodate the learning needs outlined above and the haptic interaction presented several challenges that needed to be addressed in order to minimise the "extraneous cognitive load" (Sweller, 2010). First, cell membranes and the ways in which they control the movement of substances into out of cells are extremely complex. Using real images, for example, would have been not only technically extremely difficult but also unhelpful for learning because the massive number and variety of structures would increase extraneous cognitive load and confuse the students. Therefore, it was necessary to identify suitable iconic ways of representing structure and function. Key considerations for the design of the model are discussed below but can be summarised as:

Level of complexity-Identifying a level of complexity that would be sufficiently accurate not to lead to misconceptions while being feasible to be modelled in a VR environment and not too complex for students to understand nor to generate unnecessary extraneous cognitive load.

Scale considerations - Representing the relative size and scale of structures so that they would be manipulable within a confined space given that the workspace dimensions of the haptic device with multi-finger manipulation were restricted to $30 \mathrm{~cm}$ along the $\mathrm{x}$-axis, $23 \mathrm{~cm}$ along the $\mathrm{y}$-axis and $40 \mathrm{~cm}$ along the $\mathrm{z}$-axis.

Haptic feedback - Modelling the haptic forces so that students would be able to feel forces in a way that might help them to understand their movement as well as supporting them to manipulate the model.

As mentioned previously, students have difficulties in understanding scale and magnification. Therefore, consideration was given to enabling students to navigate through a virtual cell model and negotiate scale changes. However, technical considerations in implementing such complex software meant that instead the model was restricted to a small piece of the plasma membrane. In order to aid students' comprehension of how this small piece would fit into the cell membrane, it was 


\section{A section through a liver cell (animal cell)}

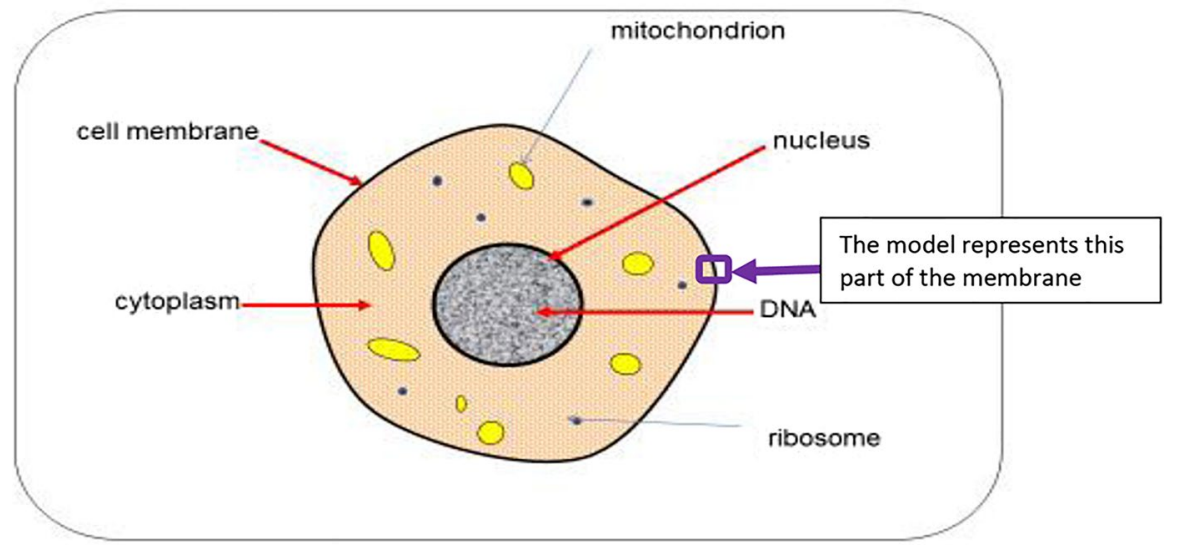

Fig. 1 Depiction on the worksheet of the cell membrane model in relation to a typical diagram of the cell

depicted on the worksheet on the type of cell diagram commonly found in textbooks as shown in Fig. 1.

Representing the elements of the model to scale also presented significant challenges. For the purposes of this investigation, approximate sizes are adequate but it is apparent, from Table 1, that representing molecules for manipulation in the VR environment with the cell membrane requires compromise on scale. For example, if an oxygen molecule is represented at the size of a blueberry $(\sim 1 \mathrm{~cm})$, representing the cell membrane to scale would make it $30 \mathrm{~cm}$ thick and this was therefore not feasible given the workspace dimensions.

The details of the molecular bilayer of the cell membrane were beyond the curriculum requirements. Therefore, in order to represent the molecules to be manipulated approximately to scale in relationship to each other, we compromised on representing the membrane as a relatively thin straw-coloured barrier with some hexagonal shapes indicating that the membrane consists of many separate molecules. The phospholipid bilayer is also indicated by the cross-sectional view depicting the arrangement of hydrophilic phosphate heads and hydrophobic

Table 1 Comparison of typical sizes of relevant structures

\begin{tabular}{lll}
\hline & $\begin{array}{l}\text { Typical Approximate Measure- } \\
\text { ments }\end{array}$ & $\begin{array}{l}\text { Approximate aver- } \\
\text { age dimension in } \\
\text { metres }\end{array}$ \\
\hline Cheek epithelial cell (diameter) & $50-80 \mu \mathrm{m}$ & $6 \times 10^{-5}$ \\
Plasma Membrane (thickness) & $6-9 \mathrm{~nm}$ & $7 \times 10^{-9}$ \\
Membrane protein & $3-6 \mathrm{~nm} \mathrm{20-110} \mathrm{nm}$ & $4 \times 10^{-9}$ to $60 \times 10^{-9}$ \\
Glucose molecule (diameter) & $1 \mathrm{~nm}$ & $1 \times 10^{-9}$ \\
Oxygen molecule/ Sodium ion & $0.1-0.5 \mathrm{~nm}$ & $1 \times 10^{-10}$ \\
\hline
\end{tabular}




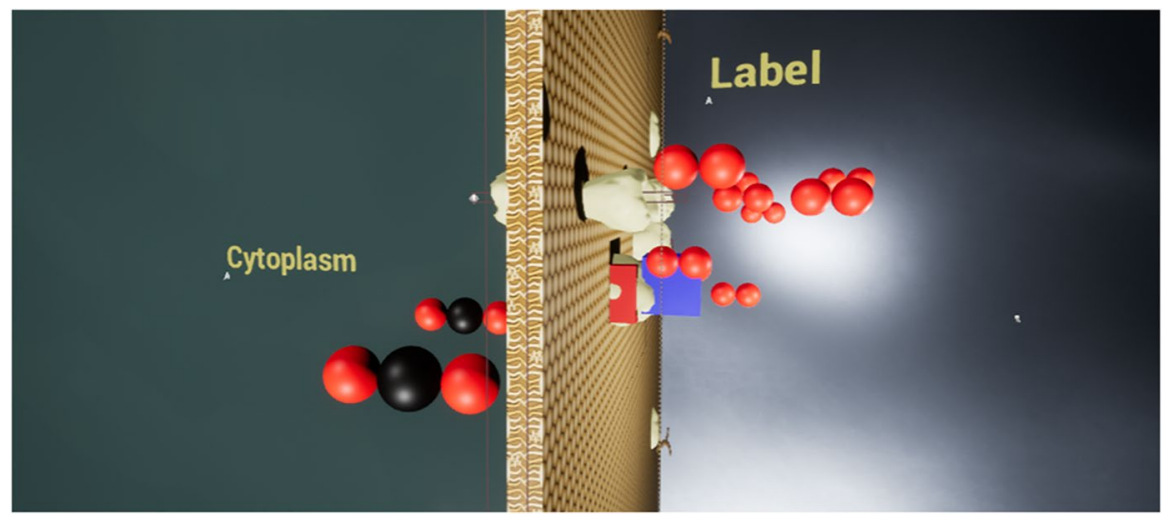

Fig. 2 View of part of the cell membrane model

tails. The screenshot in Fig. 2 shows the cell membrane model near the start of the activities.

The irregular-shaped structures penetrating through the membrane represent the membrane proteins of which several different types were modelled. Some of the membrane proteins are modelled as glucose transporters based on the GLUT1 transporter (Deng et al., 2014). As shown in Table 2, particles were represented, as far as possible, by their coloured atoms, following the CPK (Corey, Pauling, Koltun) colouring convention. For example, carbon dioxide and oxygen molecules can be seen in Fig. 2.

Modelling the movement and forces presents challenges. If the particles were modelled moving at a speed representative of their usual motion, they would be moving much too fast for the students to be able to grasp individual molecules. However, modelling them at slow speed could lead to misconceptions. Therefore, control was given to the user to move between fast, medium and slow motion. In fast motion students could feel vibrations of the molecules. In order to grasp individual molecules in order to fill forces acting on them, the model could be set to slow motion.

Table 2 Particles incorporated into the model

\begin{tabular}{lll}
\hline Particle & Colour & Type of model \\
\hline Oxygen & Red & Space filling \\
Carbon & Black & Space filling \\
Potassium ion & Dark purple & Space filling \\
Sodium ion & Light purple & Space filling \\
Glucose molecule & Light blue & Approximate shape \\
& & without atomic \\
& & detail \\
\hline
\end{tabular}




\section{The interactive learning environment and activities}

The user is able to interact with the system via two points of contact of the thumb and index finger on the same hand (either left or right), represented as blocks in the model (see Fig. 2). The interface uses a thimble device shown in Fig. 3. In the model, the finger and thumb are able to move freely through the cell membrane but when the user grabs hold of an object in the model, such as a glucose molecule, if the haptic feedback is enabled, the user feels the object and any forces acting on that object, such as those resulting from concentration gradients. In the no-hapticfeedback condition, the user interacts with the system using the same interface, but the haptic feedback is turned off in the software, so the user must rely on visual cues to grab objects. When the user makes contact with one of the substances, the 'Label' changes to show the name of the substance (carbon dioxide, oxygen, glucose, sodium and potassium). When haptic feedback is enabled, the user can feel forces on the substances, as kinaesthetic force feedback at their fingertip, depending on the concentration gradient, as they move a molecule or ion. During the simulation, users can add more molecules and ions, thus changing the concentration gradient. When a user pushes a glucose molecule towards a glucose transporter, in the haptic-feedback condition, the user feels the force as the molecule is drawn into the transporter protein and the model simulates the glucose transporter changing shape as it transports the glucose molecule through the membrane.

As shown in Fig. 4 students worked in pairs, where one student (the pilot) was immersed in the VR environment using the interface and the head-mounted display, while the other student (co-pilot) watched the interaction on a standard computer screen. The students swapped roles halfway through the activities. The pilot controlled the interaction within the VR environment while the co-pilot directed

Fig. 3 The haptic interface

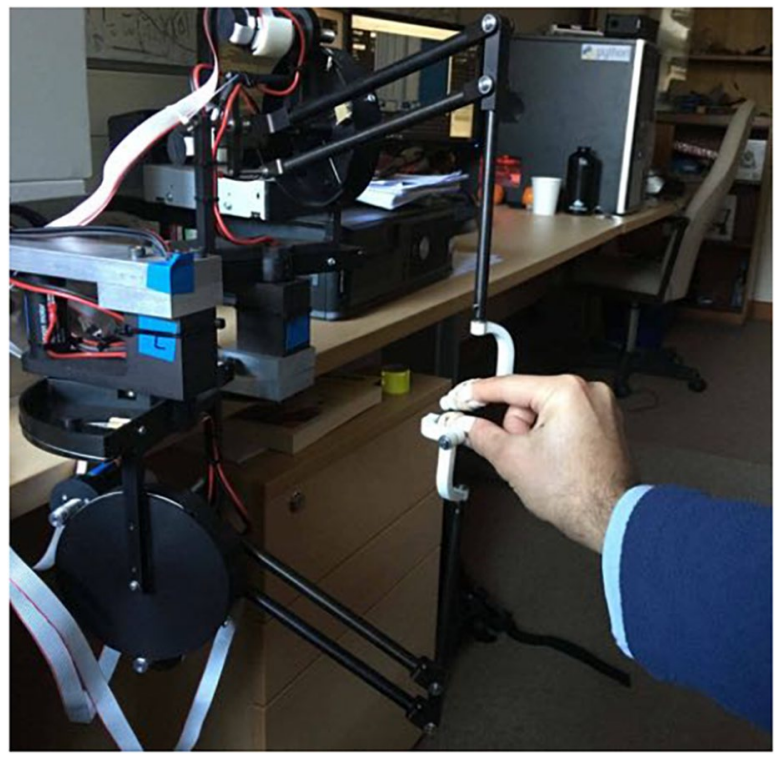




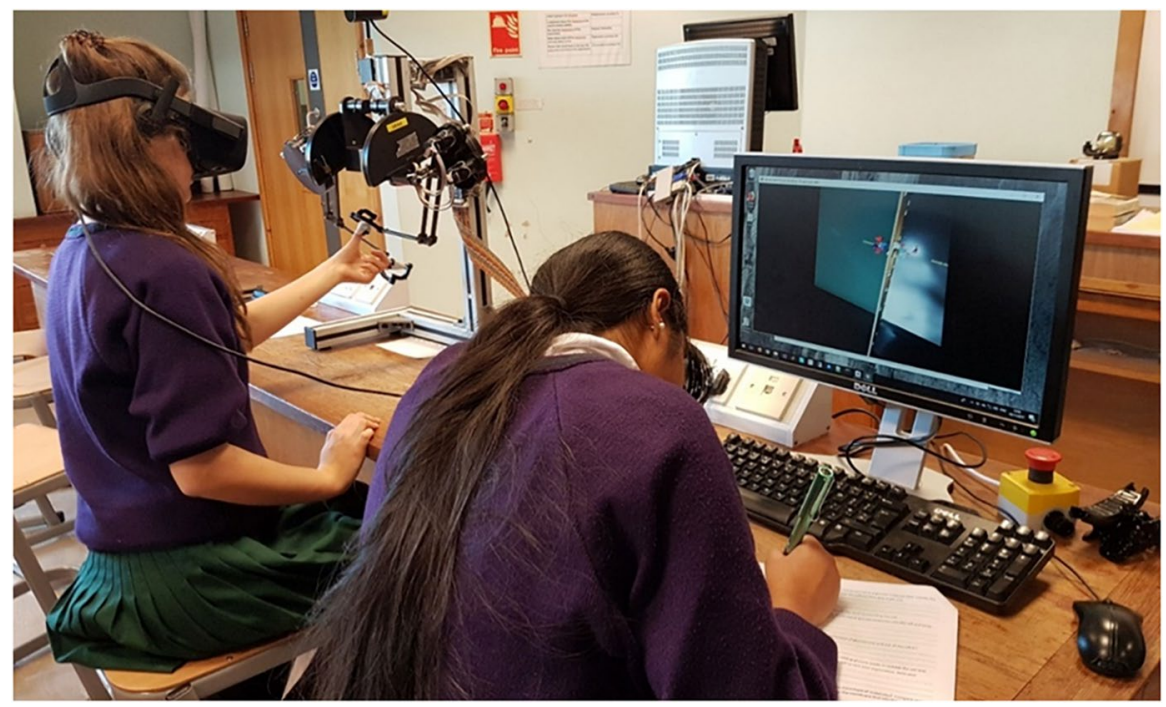

Fig. 4 Students using the system

the activities by: reading the instructions and questions on the worksheet; controlling some aspects of the model through the keyboard and writing the answers onto the worksheet. In order to focus attention on the learning objectives and the haptic feedback, and to provide scaffolds and prompts (McElhaney et al., 2015), the worksheet activities directed the pilot to perform a series of actions on objects in the model while observing visually and feeling how they moved: 1) moving membrane channel proteins within the membrane; 2) observing the movement of coloured particles; 3) touching and grabbing oxygen and glucose molecules; 4) moving an oxygen molecule into the cell and 5) moving a glucose molecule into the cell. The worksheet also instructed the students to predict changes and discuss their ideas. Thus the worksheet instructions, the activities and the specific roles, were designed to encourage collaborative learning and thus create a collective working memory (Kirschner et al., 2009), as well as to focus students on the haptic forces. The worksheets were also designed to enable students to work through the activities without teacher support which, while not typical of a classroom situation, was essential to standardise conditions for the experiment.

The design of this learning environment was informed by a series of investigations with previous prototypes and discussions with teachers and students (Webb et al., 2017). In summary, the following were the main design principles for the activities:

1. to focus students' attention on the key concepts to be learned by gradually increasing complexity in order to optimise intrinsic cognitive load (Sweller, 2016).

2. to focus students' attention on the haptic feedback in the model and the feel of the structures and processes through the activities and questions on the worksheet. 
3. to encourage students to learn collaboratively by discussing their ideas thus enabling collective working memory (Kirschner et al., 2009).

\section{Research methodology}

The study was carried out in a boys' school and a girls' school with students who were in Year 8 (aged 12-13). Both schools were independent and selective, so the students were of relatively high academic ability. Opportunist sampling was used, based on which students could be freed from lessons at the time of the study, which took place in a school laboratory away from students' classes. Pairs of students were assigned randomly to the two conditions i.e. with and without haptic feedback and the students were not informed of this difference. Data was obtained from 32 pairs of students: 16 in the haptic-feedback condition, 16 in the no-haptic-feedback condition. The procedures of the study were explained to the students and they were introduced to the systems and given assistance to position the haptic device and start the software. Our previous pilot studies had shown that students were able to use the system without training so the students embarked directly on the learning activities thus reducing the time needed for the study.

A test of biology knowledge, based on the key concepts listed above, was administered before and after the VR activities. In order to check for knowledge retention the test was administered again after a further eight months. The test consisted of three parts:

Part 1: the student was asked to write 5 important facts about the cell membrane using the following words: active transport, diffusion, permeable, oxygen, carbon dioxide, glucose, sodium ions, potassium ions, membrane proteins, channel, respiration.

Part 2: the student was asked to explain why the cell membrane is important for cells in the human body.

Part 3: the student was given 14 True/False/Unsure questions which measure knowledge of the cell membrane, diffusion and aerobic respiration.

The tests were marked and scored anonymously by a biology lecturer who was not involved in the trials and therefore had no knowledge of the students.

While students were undertaking the VR activities (approx. $40 \mathrm{~min}$ ) they were video recorded and observed by members of the research team who later compared notes and reviewed the videos in order to identify any issues in the use of the system and activities. Following the activities, we conducted semi-structured interviews with students in pairs to elicit their perspectives. Transcripts of the interviews were subjected to thematic analysis using inductive coding by two independent researchers based on a process of negotiated agreement (Campbell et al., 2013) which reached $96 \%$ agreement. 


\section{Results}

Observations showed that with or without haptic feedback students quickly became familiar with the system and were able to use it to perform the activities. Furthermore, most pairs worked well together to support each other in interacting with the model and answering the questions. Only two of the students had previously used VR systems and therefore the experience was novel. Here, we examine findings regarding knowledge gains from analysis of the knowledge tests and use students' perspectives from the interview data to help to illuminate the quantitative findings.

\subsection{Learning gains from the activities}

Overall results from the knowledge tests are shown in Table 3. Assumptions for the use of a $2 \times 3$ mixed analysis of variance (ANOVA) were met: the Kolmogorov-Smirnov test showed that the data was normally distributed $(p>0.05)$; Levene's test showed the assumption of homogeneity of variance was met for the pre-test $(F=0.057, p=0.81)$, post-test $(F=0.04, p=0.84)$ and retention $(F=0.12, p=0.726)$ scores and Mauchly's test also indicated that the assumption of sphericity was met: $\chi 2(2)=84, p=0.66)$. The $2 \times 3$ mixed ANOVA showed a significant main effect of time across time points (pre, post and retention): $F(1,104)=26.56, p<0.001$. Furthermore, post-hoc tests using the Bonferroni correction indicated that the mean score for the pre-tests $(M=24.30, S D=5.83)$ was significantly different from the mean score of the post-tests $(M=31.17, S D=6.61$. $)(p=<0.01)$ and the means for the pre-test scores were also significantly different from the retention-test scores $(M=29,63, S D=7.62)(p<0.01)$. There was no significant difference between the means of post-test and retention scores $(p=0.50)$. These results suggest that students had improved their understanding by undertaking the activities and had retained that understanding over a period of eight months.

However, whether or not the participants received haptic feedback did not affect the change in score: $\mathrm{F}(2,104)=2.42, p=0.09$. More specifically, a one-way ANOVA showed that there were no significant differences in the pre-test scores for

Table 3 Comparison of pre-, post- and retention test scores for the knowledge test

\begin{tabular}{lllll}
\hline Descriptive Statistics & & & \\
\hline & Condition & Mean & Std. Deviation & N \\
\hline \multirow{2}{*}{ Pre-test scores } & Haptic-feedback & 25.45 & 4.79 & 29 \\
& No-haptic-feedback & 22.96 & 5.83 & 25 \\
& Total & 24.30 & 5.39 & 54 \\
Post-test scores & Haptic-feedback & 31.72 & 6.07 & 29 \\
& No-haptic-feedback & 30.52 & 7.26 & 25 \\
& Total & 31.17 & 6.61 & 54 \\
Retention scores & Haptic-feedback & 28.79 & 7.89 & 29 \\
& No-haptic-feedback & 30.60 & 7.34 & 25 \\
& Total & 29.63 & 7.62 & 54 \\
\hline
\end{tabular}


haptic $(M=25.19, S D=5.40)$ and non-haptic $(M=22.56, S D=5.82)$ conditions; $\mathrm{F}(1,62)=3.45, p=0.066$. There was no significant difference in the post-test scores for haptic $(M=31.25, S D=6.03)$ and non-haptic $(M=30.69, S D=6.68)$ conditions: $\mathrm{F}(1,62)=0.13, p=0.73$.

There were also no significant differences in the retention scores between haptic $(M=28.79, S D=7.89)$ and non-haptic conditions $(M=30.60, S D=7.34): \mathrm{F}(1$, $52)=0.75, p=0.39$. Therefore, this one-way ANOVA showed that the scores in the pre-test, post-test and retention-test did not differ significantly between the haptic and non-haptic groups.

\subsection{Student perspectives on learning with the system}

Themes emerging from the student interview data, summarised in Table 4, revealed that all students believed they gained a better understanding through using the VR. Students valued the visual experience but also commented on the value of being able to grasp and move the objects. Students described how using the system helped them to visualise and build a better picture of the cell membrane in their minds:

'you can actually, like, feel it and know what it's like. Um, I learnt, if I was to do this instead of my average reading textbooks, I'd rather do this because I picked up so much more so much more quickly cos I actually saw it in my brain happen' Enzo (haptic-feedback)

'If you just learn about it without VR or anything like that you can only just imagine it. So, you can't get like a proper sense or anything. But with VR, um, as you can feel it you can get a much, um, you can get a better sense, like, you can actually imagine it properly.' - Jerome (no-haptic-feedback)

Some of the students commented specifically on the haptic feedback helping them to visualise but most of the comments focused on seeing and interacting with the models. Furthermore, the majority of statements coded to the visualisation theme came from students in the no-haptic-feedback condition.

\subsection{Collaborative learning}

Nearly all the students reported that they enjoyed working in pairs in these activities and found learning collaboratively was well supported by the system. There was no clear difference between those in the haptic-feedback compared with the no-hapticfeedback condition. Students particularly valued the communication and discussion that they believed enabled their learning (69 data items from 25 out of 32 separate interviews) in working out answers and making sense of the material. For example:

'Cause like I was kind of struggling for ideas, so then, like, Serena also had ideas, so Serena, when Serena said something, that kind of inspired me to think of something else. So that was good.' - Adalyn (haptic-feedback) 


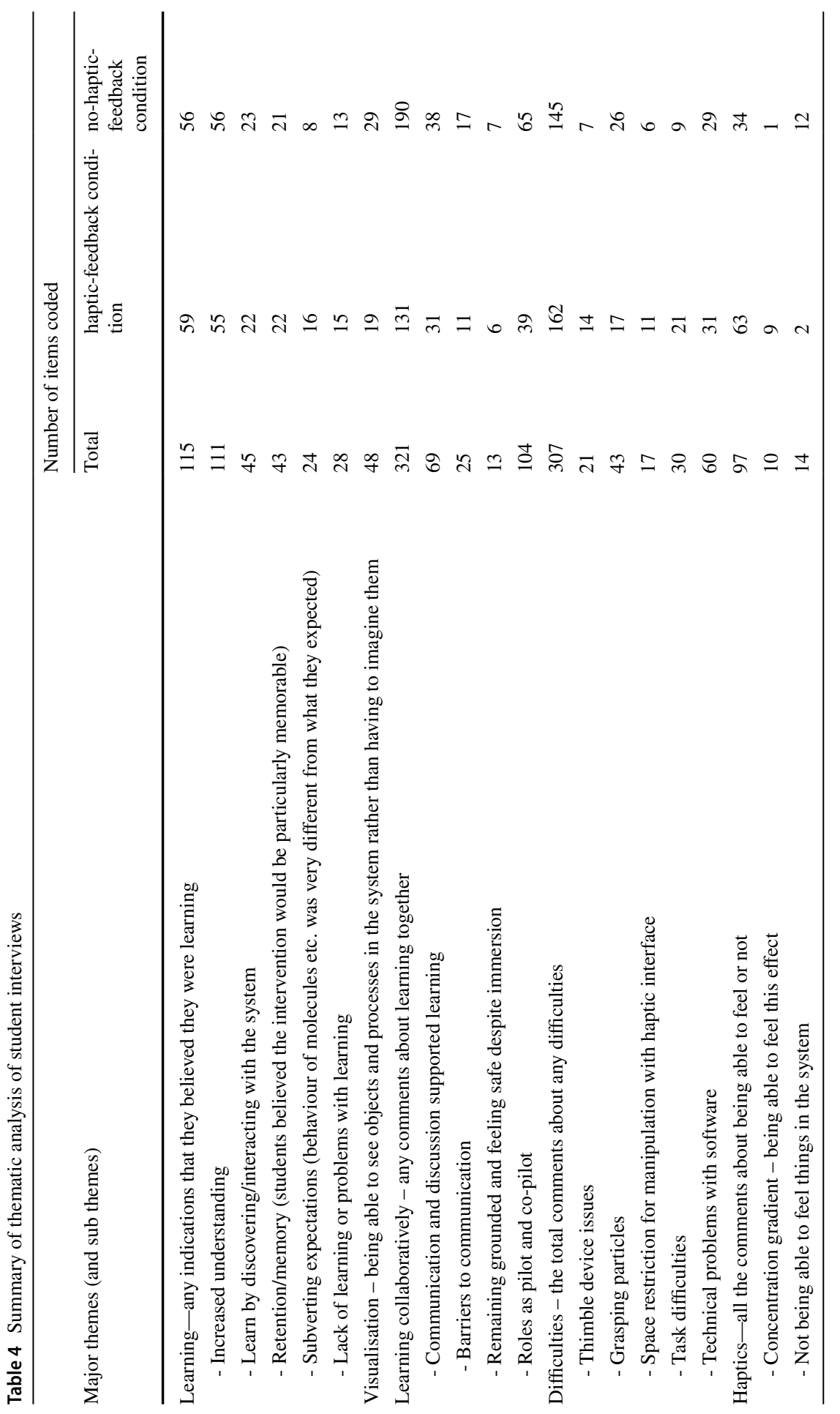


'It gives two people's perspectives. I think that always helps because then you can, almost as a team, you can come up with an answer instead of just working on your own and it's your opinion, you can combine each other's, and in the end it's probably a more accurate answer.' - Hayden (no-haptic-feedback)

In addition, the setup of the system with the co-pilot in the real world enabled the pilot in the VR environment to feel grounded and safe because with the VR headset on students reported that they felt immersed and perhaps slightly vulnerable e.g.:

'It's just having Natasha next to me just made me feel like... It just sounds weird to say but just like safer, I guess. It was just nicer to have someone next to you.' Rose (no-haptic-feedback).

\subsection{Haptic feedback effects}

As explained earlier, while the movement and interaction of particles in the model could generally be observed visually so haptic feedback was not crucial, effects of concentration gradient could only be determined by haptic feedback. Based on answers to questions regarding concentration gradient (see Table 5) the intervention was unsuccessful in developing students' understanding of passive diffusion as affected by concentration gradient. For example, regarding the movement of oxygen, although 13 students did answer correctly having previously been incorrect, 13 were still incorrect or unsure and a further 13 answered incorrectly having previously been correct. The haptic effects were perhaps too weak for the students to feel the effects of concentration gradient or perhaps other information, particularly visual, was dominating their cognitive processing.

Some students did discuss the resistance from the concentration gradient when moving molecules across the membrane (10 data items from 6 separate interviews). For example:

Yeah, the glucose was really interesting and the more you had, the more resistance the cell membrane gave. So... Yeah, that was good, I liked feeling that.' - Mikayla (haptic-feedback)

'When it was balanced on each side and imbalanced and it was harder to move them through, like so how the resistance can change depending on how many there are outside or inside the cell.' - Declan (haptic-feedback)

Therefore it was clear that some students were feeling the haptic forces. However, it was clear from the interview data that the concentration gradient haptic feedback was not always noted or discussed as frequently as other haptic feedback (e.g. the weight/ hardness of molecules and the membrane). This lack of attention to the effects of concentration gradient by many of the students may explain why the predicted benefit of haptic feedback was not found. Reasons for this failure to attend to the concentration gradient force include the possibility that some students were focused on visual effects as predicted by the visual dominance theory.

Some students, especially in the no-haptic-feedback condition, found difficulty in grasping the objects in the system (see Table 4). Therefore, while generally haptic 
Table 5 Answer change between pre-and post-tests - concentration gradient

Statement: If there is an equal amount of oxygen inside and outside the cell it will be harder for oxygen to enter than if there is more oxygen outside

\begin{tabular}{lll} 
& \multicolumn{2}{l}{ No. of students responding } \\
Answer change pre-post & Haptic-feedback & No-haptic-feedback \\
No change-correct & 10 & 9 \\
No change-incorrect & 1 & 3 \\
No change-unsure & 3 & 5 \\
Correct to incorrect & 3 & 4 \\
Correct to unsure & 3 & 3 \\
Incorrect to correct & 2 & 2 \\
Incorrect to unsure & 4 & 5 \\
Unsure to correct & 4 & 0
\end{tabular}

Statement: If there is an equal amount of carbon dioxide inside the cell and outside the cell it will be harder for carbon dioxide to leave the cell than if there is more carbon dioxide outside

\begin{tabular}{lll} 
Answer change pre-post & Haptic-feedback & No-haptic-feedback \\
No change-correct & 9 & 2 \\
No change-incorrect & 1 & 0 \\
No change-unsure & 3 & 5 \\
Correct to incorrect & 7 & 2 \\
Correct to unsure & 2 & 1 \\
Incorrect to correct & 1 & 1 \\
Unsure to correct & 4 & 10 \\
Unsure to incorrect & 6 & 4 \\
\hline
\end{tabular}

feedback was not essential for students to manipulate the model, some students did find such manipulation difficult and were therefore hindered by the lack of haptic feedback.

\subsection{Factors affecting extraneous cognitive load}

Of the more than 300 data items concerning difficulties only 30 were about difficulties with the activities. Students' comments showed that system problems were twice as prevalent in the haptic-feedback compared with the no-haptic-feedback condition (See Table 4) and this was confirmed by observations. Thus, there were more interruptions in the haptic-feedback condition mainly because of the vibrations of the molecules causing juddering of the haptic interface which sometimes dislodged the thimble devices on small fingers. These problems were quite quickly resolved by technicians or the students themselves and generally did not interfere significantly with the progress of activities. However, these distractions would have contributed to the extraneous cognitive load on the students in the haptic-feedback condition thus probably detracting from any decrease in cognitive load created by 
the synergistic effects predicted by dual coding theory. Technical problems with the software caused minor disruptions and were equally prevalent in both conditions.

\section{Discussion and Conclusion}

The learning gains together with findings from the student interviews suggest that the design of the 3D VR model and interface and the associated activities were: suitable for the target students; enabled collaborative learning through the pilot and copilot model of interaction; were motivating and supported students in learning most of the key concepts.

As there was no significant difference in knowledge gains between the two conditions, we can conclude that the haptic feedback, in spite of increased technical problems especially juddering and the need to restart the system fairly frequently, did not adversely affect learning. On the other hand, the haptic feedback was not essential for most students to interact effectively with the system. Observation of the students while they were undertaking the activities as well as students' own perspectives revealed that turning off the haptic feedback generally did not inhibit most of the students from interacting with the system and carrying out the activities. Therefore, they were able to compensate for the lack of haptic feedback through visual cues. Therefore, we can conclude that the haptic feedback was not essential for the learning of those concepts for the majority of students in this study. However, a minority of students did find grasping the particles difficult in the absence of haptic feedback.

The design of the learning systems and activities was expected to create a high intrinsic cognitive load for these students because the activities addressed a range of interrelated concepts and the pre-tests showed that most students started with limited understanding. Furthermore, the results suggest that the extraneous cognitive load was also contributing to a high cognitive load environment. According to Cognitive Load Theory (Sweller, 2010) students' learning is limited by their working memory which is subject to a maximum cognitive load. Intrinsic and extraneous cognitive load are additive such that extraneous cognitive load limits the potential for intrinsic cognitive load that is able to be processed in working memory and thus to enable learning (Sweller, 2016).

Observations during activities suggested that some students were not experiencing the full effects of the haptic feedback that we had expected. This may have been because the haptic stimuli were not strong enough for students to notice because the visual stimuli dominated their attentional systems in this high cognitive load environment. As discussed earlier, studies have shown that the visual dominance effect can be overcome by "user priming" or through task instruction (Pusch \& Lécuyer, 2011). Therefore, the possibility remains that learning of some concepts might be supported by haptic feedback if the haptic feedback were strengthened or if students' attention were focused more specifically on haptic feedback. Therefore, when deploying such activities in a normal classroom setting, the teacher's role could include focusing the students on the haptic feedback where appropriate through 
scaffolding, questioning, explanations and discussion. These possibilities are worthy of further research.

Based on findings from this study and others, haptics has yet to demonstrate sufficient utility for educational use, although the technology is still evolving. In contrast VR that is accompanied with strong links between the movements of the physical fingers and the simulation of the fingers shows strong promise. Whether or not the haptic feedback is critical for students' learning, the addition of haptic feedback to a VR system does provide a more complete and authentic experience. Students reported that being able to grasp objects without the sense of touch felt strange. Furthermore, in this study, the haptic feedback did not adversely affect learning even though some technical problems were encountered. If, as expected, it becomes possible to provide relatively inexpensive haptic interfaces to VR systems, then our findings suggest that incorporating haptic feedback would provide a more authentic experience for all and might be necessary to enable some students to interact effectively with the system. We cannot be certain from our findings whether or not all the pedagogical elements were crucial for learning, but our analysis of students' perspectives suggested that watching the processes in 3D, interacting with the system by manipulating objects and collaborative interaction and discussion with other students all supported their learning.

Collaboration including discussion was valued by the students for enabling their learning and this should be considered in designing simulations. Enabling collaborative learning in immersive VR environments presents challenges. Typically, an individual student is quite isolated by wearing a VR headset. In this study, the roles of pilot and co-pilot and the arrangement of the VR system enabled collaborative learning as well as making the student who was immersed in the environment also feel grounded in the real world. Thus, our findings suggest that this pilot/co-pilot model of collaborative learning is worthy of further research for using 3D VR environments in schools. This approach builds on a similar paired learning process, "pair programming", which as mentioned earlier has been used successfully in schools. This model uses the roles of pilot/navigator where one of the pair (the pilot) is writing the program on the computer while the other (the navigator) provides guidance and feedback. In the current study we did initially, in our pilot studies, designate roles as pilot and navigator but our observations suggested that, in this context where navigation around a virtual cell was actually necessary, students in the roles of navigators focused too much on finding their way around the system rather than taking a more equal share in solving problems generally. Therefore, we believe that the pilot/co-pilot model provides the most promising opportunity for collaborative learning in a VR environment. Students in the current study identified the value of sharing ideas and problems but further research is needed to examine in detail its effects on outcomes. Further research might also compare this model with one in which both students use VR headsets and are therefore fully immersed. However, our findings suggest that having one student immersed and the other grounded in the real world provides a powerful collaborative learning environment that enables dialogue and exchange of ideas.

The design of the model required compromise, particularly regarding modelling of relative sizes of the nanoscale structures, owing to space limitations imposed by the haptic interface. These limitations are difficult to overcome with current technology. 
Furthermore, in order to appreciate the haptic forces on molecules, the membrane model was much simplified: our model imposed no resistance when the cursors moved through the lipid bilayer which presented as a static structure. Modelling the lipid bilayer more accurately as a dynamic structure would present significant challenges for programming the haptic feedback. However, a possibility for modelling fluidity could be achieved, with more resources, by superimposing simulation of the dynamic visual appearance of membrane fluidity.

Alternatively, or in addition to these software improvements, there is a role for teachers in mediating discussions and scaffolding the students to support their comparison of the model with reality as well as in understanding scale changes and different levels of representation (Olander et al., 2018). In the current study, in order to control the learning environment as much as possible to enable comparison of the haptic-feedback with nohaptic-feedback conditions, there was no interaction between students and their teacher. However, when activities are integrated into classroom learning teachers will have an important mediating and scaffolding role. It is expected that similar activities could be incorporated into normal classroom lessons. For example, haptic activities might be incorporated into a circus of investigations, in which students move around from one activity to the next during a lesson. Such activities might include various investigations using VR and haptic feedback as well as the standard laboratory cell studies.

Acknowledgements The authors thank the participants for their valuable feedback and are pleased to acknowledge support for this work from The Leverhulme Trust for the project `3D Learning in a Rich, Cooperative Haptic Environment'. We are also pleased to thank our colleagues on this project Jon Rashid, Carleen Houbart, Phil James, Richard Fisher, Simon Bliss, Peter Tolson and Balazs Janko.

Open Access This article is licensed under a Creative Commons Attribution 4.0 International License, which permits use, sharing, adaptation, distribution and reproduction in any medium or format, as long as you give appropriate credit to the original author(s) and the source, provide a link to the Creative Commons licence, and indicate if changes were made. The images or other third party material in this article are included in the article's Creative Commons licence, unless indicated otherwise in a credit line to the material. If material is not included in the article's Creative Commons licence and your intended use is not permitted by statutory regulation or exceeds the permitted use, you will need to obtain permission directly from the copyright holder. To view a copy of this licence, visit http://creativecommons.org/licen ses/by/4.0/.

\section{References}

Anmarkrud, Ø., Andresen, A., \& Bråten, I. (2019). Cognitive load and working memory in multimedia learning: Conceptual and measurement issues. Educational Psychologist, 54(2), 61-83. https://doi. org/10.1080/00461520.2018.1554484

Bivall, P., Ainsworth, S., \& Tibell, L. A. E. (2011). Do haptic representations help complex molecular learning? Science Education, 95(4), 700-719. https://doi.org/10.1002/sce.20439

Broadbent, H. J., Osborne, T., Rea, M., Peng, A., Mareschal, D., \& Kirkham, N. Z. (2018). Incidental category learning and cognitive load in a multisensory environment across childhood. Developmental Psychology, 54(6), 1020-1028. https://doi.org/10.1037/dev0000472

Burton, H., \& Sinclair, R. J. (2000). Attending to and remembering tactile stimuli: A review of brain imaging data and single-neuron responses. Journal of Clinical Neurophysiology, 17(6), 575-591. https://journals.1ww.com/clinicalneurophys/Fulltext/2000/11000/Attending_to_and_Remembering_ Tactile_Stimuli_A.4.aspx. 
Campbell, J. L., Quincy, C., Osserman, J., \& Pedersen, O. K. (2013). Coding in-depth semistructured interviews: Problems of unitization and intercoder reliability and agreement. Sociological Methods \& Research, 42(3), 294-320. https://doi.org/10.1177/0049124113500475

Chen, J., Wang, M., Kirschner, P. A., \& Tsai, C. C. (2018). The role of collaboration, computer use, learning environments, and supporting strategies in CSCL: A Meta-Analysis. Review of Educational Research, 0034654318791584. https://doi.org/10.3102/0034654318791584.

Deng, D., Xu, C., Sun, P., Wu, J., Yan, C., Hu, M., \& Yan, N. (2014). Crystal structure of the human glucose transporter GLUT1. Nature, 510, 121. https://doi.org/10.1038/nature13306

Flores, F., Tovar, M. E., \& Gallegos, L. (2003). Representation of the cell and its processes in high school students: An integrated view. International Journal of Science Education, 25(2), 269-286. https:// doi.org/10.1080/09500690210126793

Gallace, A., \& Spence, C. (2009). The cognitive and neural correlates of tactile memory. Psychological Bulletin, 135(3), 380-406. https://doi.org/10.1037/a0015325

Garvin-Doxas, K., \& Klymkowsky, M. W. (2008). Understanding randomness and its impact on student learning: Lessons learned from building the Biology Concept Inventory (BCI). CBE Life Sciences Education, 7(2), 227-233. https://doi.org/10.1187/cbe.07-08-0063

Gibson, J. J., \& Carmichael, L. (1966). The senses considered as perceptual systems (Vol. 2): Houghton Mifflin Boston.

Gilbert, J. K. (2005). Visualization: A metacognitive skill in science and science education. In Visualization in science education (pp. 9-27): Springer.

Gilbert, J. K., Reiner, M., \& Nakhleh, M. (2007). Visualization: Theory and practice in science education (Vol. 3): Springer Science \& Business Media.

Harwin, W., \& Barrow, A. (2013). Multi-finger grasps in a dynamic environment. In Multi-finger Haptic Interaction (pp. 5-30): Springer.

Jones, M. G., Minogue, J., Tretter, T. R., Negishi, A., \& Taylor, R. (2006). Haptic augmentation of science instruction: Does touch matter? Science Education, 90(1), 111-123. https://doi.org/10.1002/ sce. 20086

Kirschner, F., Paas, F., \& Kirschner, P. A. (2009). A cognitive load approach to collaborative learning: United brains for complex tasks. Educational Psychology Review, 21(1), 31-42. https://doi.org/10. 1007/s10648-008-9095-2

Kirschner, P. A., Sweller, J., Kirschner, F., \& Zambrano, R. J. (2018). From cognitive load theory to collaborative cognitive load theory. International Journal of Computer-Supported Collaborative Learning, 13(2), 213-233. https://doi.org/10.1007/s11412-018-9277-y

Lederman, S. J., \& Klatzky, R. L. (2009). Haptic perception: A tutorial. Attention, Perception, \& Psychophysics, 71(7), 1439-1459. https://doi.org/10.3758/APP.71.7.1439

Linn, M. C., \& Petersen, A. C. (1985). Emergence and characterization of sex differences in spatial ability: A meta-analysis. Child Development, 56(6), 1479-1498. https://doi.org/10.2307/ 1130467

Lukas, S., Philipp, A. M., \& Koch, I. (2010). Switching attention between modalities: Further evidence for visual dominance. Psychological Research PRPF, 74(3), 255-267. https://doi.org/10.1007/ s00426-009-0246-y

Malińska, L., Rybska, E., Sobieszczuk-Nowicka, E., \& Adamiec, M. (2016). Teaching about water relations in plant cells: An uneasy struggle. CBE-Life Sciences Education, 15(4). https://doi.org/10. 1187/cbe.15-05-0113

Marsh, G., Parkes, T., \& Boulter, C. (2001). Children's understanding of scale-the use of microscopes. School Science Review, 82(301), 27-31.

Mayer, R. E., \& Moreno, R. (2003). Nine ways to reduce cognitive load in multimedia learning. Educational Psychologist, 38(1), 43-52. https://doi.org/10.1207/S15326985EP3801_6

McElhaney, K. W., Chang, H.-Y., Chiu, J. L., \& Linn, M. C. (2015). Evidence for effective uses of dynamic visualisations in science curriculum materials. Studies in Science Education, 51(1), 49-85. https://doi.org/10.1080/03057267.2014.984506

McKnight, S., Melder, N., Barrow, A., Harwin, W. S., \& Wann, J. (2005). Perceptual cues for orientation in a two finger haptic grasp task. Paper presented at the First Joint Eurohaptics Conference and Symposium on Haptic Interfaces for Virtual Environment and Teleoperator Systems. World Haptics Conference. https://doi.org/10.1109/WHC.2005.107.

Melder, N., \& Harwin, W. S. (2004). Extending the friction cone algorithm for arbitrary polygon based haptic objects. Paper presented at the 12th International Symposium on Haptic Interfaces 
for Virtual Environment and Teleoperator Systems, 2004. HAPTICS'04. Proceedings. https:// doi.org/10.1109/HAPTIC.2004.1287201.

Merchant, Z., Goetz, E. T., Cifuentes, L., Keeney-Kennicutt, W., \& Davis, T. J. (2014). Effectiveness of virtual reality-based instruction on students' learning outcomes in K-12 and higher education: A meta-analysis. Computers \& Education, 70, 29-40. https://doi.org/10.1016/j.compedu.2013. 07.033

Minogue, J., \& Jones, M. G. (2009). Measuring the impact of haptic feedback using the SOLO taxonomy. International Journal of Science Education, 31(10), 1359-1378. https://doi.org/10.1080/ 09500690801992862

Novak, M., \& Schwan, S. (2021). Does touching real objects affect learning? Educational Psychology Review, 33(2), 637-665. https://doi.org/10.1007/s10648-020-09551-z

Oberauer, K., \& Eichenberger, S. (2013). Visual working memory declines when more features must be remembered for each object. Memory \& Cognition, 41(8), 1212-1227. https://doi.org/10. 3758/s13421-013-0333-6

Olander, C., Wickman, P. O., Tytler, R., \& Ingerman, A. (2018). Representations as mediation between purposes as junior secondary science students learn about the human body. International Journal of Science Education, 40(2), 204-226. https://doi.org/10.1080/09500693.2017. 1407464

Oztas, F. (2014). How do high school students know diffusion and osmosis? High school students' difficulties in understanding diffusion \& osmosis. Procedia - Social and Behavioral Sciences, 116, 3679-3682. https://doi.org/10.1016/j.sbspro.2014.01.822

Paivio, A. (1969). Mental imagery in associative learning and memory. Psychological Review, 76(3), 241-263. https://doi.org/10.1037/h0027272

Paivio, A. (2014). Intelligence, dual coding theory, and the brain. Intelligence, 47, 141-158. https://doi. org/10.1016/j.intell.2014.09.002

Posner, M. I., Nissen, M. J., \& Klein, R. M. (1976). Visual dominance: An information-processing account of its origins and significance. Psychological Review, 83(2), 157-171. https://doi.org/10. 1037/0033-295X.83.2.157

Pusch, A., \& Lécuyer, A. (2011). Pseudo-haptics: from the theoretical foundations to practical system design guidelines. Paper presented at the 13th international conference on multimodal interfaces. https://doi.org/10.1145/2070481.2070494.

Rodríguez, F. J., Price, K. M., \& Boyer, K. E. (2017). Exploring the Pair Programming Process: Characteristics of Effective Collaboration. Paper presented at the ACM SIGCSE Technical Symposium on Computer Science Education, Seattle, Washington, USA. https://doi.org/10.1145/3017680.3017748.

Rundgren, C. J., \& Tibell, L. A. E. (2010). Critical features of visualizations of transport through the cell membrane-an empirical study of upper secondary and tertiary students' meaning-making of a still image and an animation. International Journal of Science and Mathematics Education, 8(2), 223-246. https://doi.org/10.1007/s10763-009-9171-1

Rutten, N., van Joolingen, W. R., \& van der Veen, J. T. (2012). The learning effects of computer simulations in science education. Computers \& Education, 58(1), 136-153. https://doi.org/10.1016/j. compedu.2011.07.017

San Diego, J. P., Cox, M. J., Quinn, B. F. A., Newton, J. T., Banerjee, A., \& Woolford, M. (2012). Researching haptics in higher education: The complexity of developing haptics virtual learning systems and evaluating its impact on students' learning. Computers \& Education, 59(1), 156-166. https://doi.org/10.1016/j.compedu.2011.11.009

Schmid, C., Büchel, C., \& Rose, M. (2011). The neural basis of visual dominance in the context of audiovisual object processing. NeuroImage, 55(1), 304-311. https://doi.org/10.1016/j.neuroimage.2010. 11.051

Schönborn, K. J., Bivall, P., \& Tibell, L. A. E. (2011). Exploring relationships between students' interaction and learning with a haptic virtual biomolecular model. Computers \& Education, 57(3), 20952105. https://doi.org/10.1016/j.compedu.2011.05.013

Seifi, H., Chun, M., Gallacher, C., Schneider, O., \& MacLean, K. E. (2020). How do novice hapticians design? A case study in creating haptic learning environments. IEEE Transactions on Haptics, 13(4), 791-805. https://doi.org/10.1109/TOH.2020.2968903

Shams, L., \& Seitz, A. R. (2008). Benefits of multisensory learning. Trends in Cognitive Sciences, 12(11), 411-417. https://doi.org/10.1016/j.tics.2008.07.006

Sweller, J. (1994). Cognitive load theory, learning difficulty, and instructional design. Learning and Instruction, 4(4), 295-312. https://doi.org/10.1016/0959-4752(94)90003-5 
Sweller, J. (2010). Element interactivity and intrinsic, extraneous, and germane cognitive load. Educational Psychology Review, 22(2), 123-138. https://doi.org/10.1007/s10648-010-9128-5

Sweller, J. (2016). Working memory, long-term memory, and instructional design. Journal of Applied Research in Memory and Cognition, 5(4), 360-367. https://doi.org/10.1016/j.jarmac.2015.12.002

Tasker, R., \& Dalton, R. (2008). Visualizing the molecular world-Design, evaluation, and use of animations. In J. K. Gilbert, M. Reiner, \& M. Nakhleh (Eds.), Visualization: Theory and practice in science education (pp. 103-131). Springer. https://doi.org/10.1007/978-1-4020-5267-5_6.

Tekkaya, C. (2003). Remediating high school students' misconceptions concerning diffusion and osmosis through concept mapping and conceptual change text. Research in Science \& Technological Education, 21(1), 5-16. https://doi.org/10.1080/02635140308340

Tokatli, O., Tracey, M., Barrett, N., Hwang, F., Johnson, R., Jones, C., Macaulay, K., Webb, M E., Harwin, W. (2016). Haptic interactions to support biology education: navigating the cell. Paper presented at the Euro haptics, Imperial College.

Tuckey, H., \& Selvaratnam, M. (1993). Studies involving three-dimensional visualisation skills in chemistry: A review. Studies in Science Education, 21(1), 99-121. https://doi.org/10.1080/0305726930 8560015

Umapathy, K., \& Ritzhaupt, A. D. (2017). A meta-analysis of pair-programming in computer programming courses: implications for educational practice. ACM Trans. Comput. Educ., 17(4), Article 16. https://doi.org/10.1145/2996201

Webb, M. E. (2008). Impact of IT on science education. In J. Voogt \& G. Knezek (Eds.), International Handbook of Information Technology in Primary and Secondary Education (pp. 133-148). Springer. ISBN: 978-0-387-73314-2.

Webb, M. E., Tracey, M., Harwin, W., Tokatli, O., Hwang, F., Johnson, R., Barrett, N., Jones, C. (2017). The potential for haptic-enabled interaction to support collaborative learning in school biology. Paper presented at the Society for Information Technology \& Teacher Education International Conference 2017, Austin, TX, United States. https://www.learntechlib.org/p/177885.

Wong, A., Leahy, W., Marcus, N., \& Sweller, J. (2012). Cognitive load theory, the transient information effect and e-learning. Learning and Instruction, 22(6), 449-457. https://doi.org/10.1016/j.learninstr uc.2012.05.004

Xu, Y., \& Franconeri, S. L. (2015). Capacity for visual features in mental rotation. Psychological Science, 26(8), 1241-1251. https://doi.org/10.1177/0956797615585002

Zacharia, Z. C. (2015). Examining whether touch sensory feedback is necessary for science learning through experimentation: A literature review of two different lines of research across K-16. Educational Research Review, 16, 116-137. https://doi.org/10.1016/j.edurev.2015.10.001

Publisher's note Springer Nature remains neutral with regard to jurisdictional claims in published maps and institutional affiliations. 